\title{
Structural alterations of an ambiguous musical figure: The scale illusion revisited
}

\author{
GABRIEL A. RADVANSKY, WILLIAM M. HARTMANN, and BRAD RAKERD \\ Michigan State University, East Lansing, Michigan
}

\begin{abstract}
The scale illusion (Deutsch, 1975) shows the importance of frequency range in the perceptual organization of a sequence of notes. This paper includes three experiments on the scale illusion. Experiments 1 and 2 demonstrated that if the structure of the pattern of notes used in the original scale illusion study is altered slightly, by adding or subtracting a pair of notes from the ends of the sequence, there is a significant decrease in the rate of frequency-based responses, suggesting a weaker illusion. Experiment 3 investigated two features of the note patterns that may have led to this change. Specifically, it asked whether the decrease in the strength of the illusion is due to (1) the nature of the notes at the extremes of the frequency range and/or (2) the nature of the notes at the crossing point of the two scales. While both sources were found to affect the strength of the scale illusion, the former had a greater influence.
\end{abstract}

Frequency range is known to provide a reliable basis for the perceptual organization of complex tonal streams (Bregman \& Campbell, 1971) and to provide a strong cue that makes the task of recognizing interleaved melodies easier (Dowling, 1968, 1973; Hartmann \& Johnson, 1991). Organizing by frequency range is conceptually equivalent to organizing according to the gestalt principle of proximity. An alternative basis for the organization of notes is provided by their "tonal trajectory" (cf. Tougas \& Bregman, 1987, 1990) as manifested in such ascending or descending structures as major, minor, and chromatic scales. Such an organization represents a form of good continuation.

When put in competition, the influence of a good continuation organization does not approach the strength of proximity. Tougas and Bregman $(1987,1990)$ presented pure-tone stimuli that could be organized equally well on the basis of proximity (frequency range) or good continuation (a pattern of tones that progressively ascended or descended). Listeners unwaveringly reported proximitybased perceptions. This preference occurred even when emphasis on the tonal trajectory was given by clear ascending and descending passages prior to the critical sequence.

A third basis for organizing musical notes is by their spatial location. For example, one could group by ear of input in a dichotic listening experiment, that is, accord-

This research was supported in part by NIDCD Grant DC0081 to W.M.H. The authors would like to thank Beth Chittenden for her assistance in sorting listener responses and Rose Zacks for her helpful comments at various stages of the research. W. M. Hartmann is in the Department of Physics and Astronomy and B. Rakerd is in the Department of Audiology and Speech Sciences at Michigan State University. All correspondence regarding this article should be directed to G. A. Radvansky, who is now at the Department of Psychology, University of Illinois, 603 E. Daniel Street, Champaign, IL 61820. ing to laterality. Laterality, like good continuation, is apparently less persuasive than proximity. Deutsch (1974) showed this in an effect called the "octave illusion." She presented a dichotic sequence of notes an octave apart, with the notes in the two octaves repeatedly switching ears. When asked what was perceived in each ear, listeners did not report the alternating high and low notes that were truly presented. Instead, they reported the high notes lateralized in one ear and the low notes in the other.

Finally, proximity has been found to be the preferred organization when put in competition with both good continuation and laterality. This is the essence of an effect called the scale illusion (Butler, 1979; Deutsch, 1975; Smith, Hausfield, Power, \& Gorta, 1982). In scaleillusion experiments, listeners are asked to report on the dichotically presented note pattern illustrated in Figure 1a. This pattern is constructed from two diatonic major scales, one ascending and the other descending. The notes presented to each ear are alternately drawn from the ascending and descending scales, giving rise to "jagged" input patterns at each ear. Deutsch found that the vast majority of listeners, when asked to either verbally describe or shadow sing the input to each ear, gave reports like those illustrated in Figure 1b. Specifically, they reported hearing a high-frequency "arc" made up of a falling then a rising pattern of notes in one ear, and a low-frequency arc made up of a rising then a falling pattern of notes in the other ear. In effect, the input to the two ears was (1) separated out on the basis of frequency, (2) grouped into a pattern, and (3) "reassigned"' to the two input channels. This is quite a remarkable demonstration of the organizational persuasiveness that proximity (frequency) may have. Those few listeners who did not report the complete pattern tended to report only the high-frequency arc.

Alternatives to this proximity-based organization of the notes are shown in Figures 1a and 1c. A report of Figure la would indicate that the listeners were able to ve- 
(o)

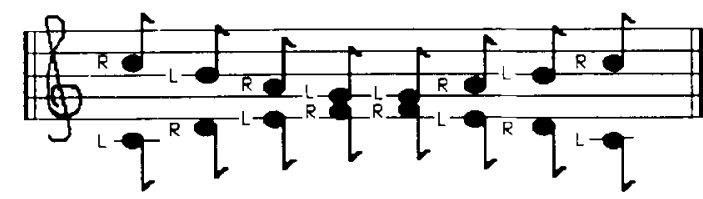

(a)

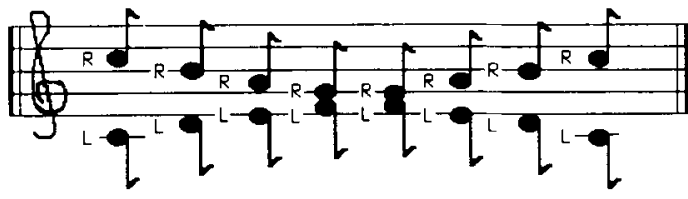

(c)

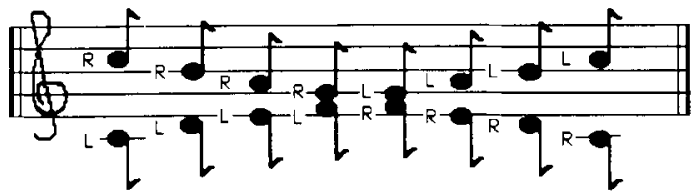

Figure 1. Examples of possible organizations of the pattern of notes presented to listeners in scale-illusion experiments. Pattern a is a veridical laterality organization, Pattern $b$ is a proximity organization, and Pattern $c$ is a good continuation organization.

ridically report what was presented to each ear, that is, to group in terms of laterality in accordance with the task. Deutsch's (1975) listeners never reported this. Figure 1c illustrates good continuation patterns that could have been constructed from the notes; one was an ascending scale and the other a descending scale. Although a report of such a pattern would require no more reassignment of the ear input than does proximity grouping, it was never reported by Deutsch's listeners, even in a single ear.

A common finding in studies of perceptual organization is that several different gestalt principles are simultaneously at work and that they, in effect, codetermine the outcome. The results of the scale-illusion experiment make it tempting to conclude that, for music perception, a single principle-grouping by proximity-is so dominant that the other principles can be greatly discounted, or ignored altogether.

However, in all of the scale-illusion experiments done to date, only a single pattern of notes has been used to assess the competing influences of proximity, good continuation, and laterality. That is the pattern illustrated in Figure 1a. The purpose of the present experiments was to determine whether the details of this pattern were somehow critical to the dominance of proximity. Specifically, we asked whether the inherent symmetry of the 8-note pattern is a major contributor.

\section{EXPERIMENT 1}

Experiment 1 replicated and extended the original work by Deutsch (1975) with a notable modification: to Deutsch's pattern (Figure 1a), which has an even number of notes $(N=8)$, were added two new patterns, each having an odd number of notes $(N=7$ and $N=9)$.

\section{Method}

\section{Listeners}

Sixteen listeners were recruited from the subject pool at Michigan State University and given partial course credit for their participation. The listeners reported playing musical instruments for $0-8$ years $(M=3.3)$ with $0-8$ years of instruction $(M=2.9)$. No listeners in any experiment reported having ear training, perfect pitch, or any hearing problems. Only 1 listener (in Experiment 3) reported having had formal music theory.

\section{Stimuli}

The note patterns for this study were of four types, as illustrated in Figure 2. The first type (Figure 2a), the pattern used by Deutsch (1975), consisted of concurrent ascending and descending C-major scales $\left(C_{4}-C_{5}\right)$, with the input to each ear alternately coming from one scale and then the other. This will be referred to as the $D$ (for Deutsch) pattern. Two other patterns were like the $D$ pattern except for the range of the scales: Pattern $D+1$ (Figure 2b) was a 9-note scale $\left(\mathrm{C}_{4}-\mathrm{D}_{5}\right)$; Pattern $\mathrm{D}-1$ (Figure $2 \mathrm{c}$ ) was a 7 -note scale $\left(\mathrm{C}_{4}-\mathrm{B}_{4}\right.$ ). The fourth pattern (Figure $2 \mathrm{~d}$ ) consisted of the two $\mathrm{C}$-major scales blocked by ear of input to eliminate ear switching (cf. Smith et al., 1982, Experiment 2). This last pattern will be referted to as the NS (no-switch) pattern. The NS pattern was included to test the dominance of proximity-based organization when good continuation and laterality cues are both arrayed against it. If frequency is an overwhelming organizer, then listeners should report proximity-based percepts for this condition as well.
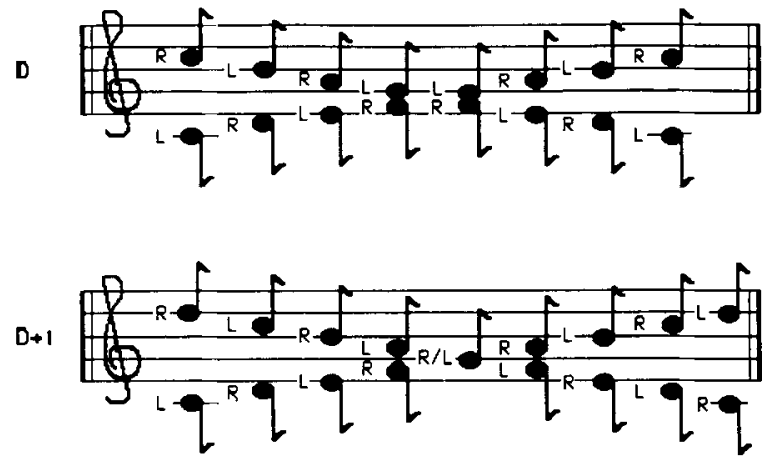

D-1

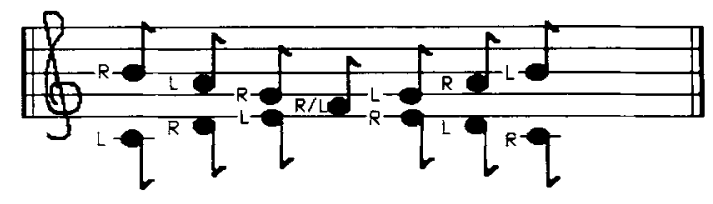

NS

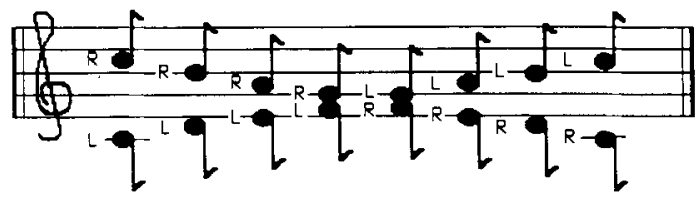

Figure 2. Examples of note patterns presented to listeners in Experiment 1. Note Pattern $D$ is the original Deutsch (1975) note pattern; Note Patterns D+1 and D-1 are altered D note patterns, and Note Pattern NS has no shift in a scale's ear of presentation. Note patterns were also presented with reversed ear of input. Experiment 2 only presented Note Patterns D and D+1. 
Each pattern type was played to the listeners in two ways: (1) with the initial high note in the right ear and (2) with the initial high note in the left ear. This was done to control for any effects of events starting in one ear or another. This will be referred to as the origin variable. On each trial, a listener heard 10 continuous repetitions of a particular note pattern. Across a block of 8 trials, all versions of the stimuli ( 4 patterns $\times 2$ forms of origin) were presented once in a random order. The entire test was composed of six such blocks, for a total of 48 trials. The only constraint was that the last note pattern of a block could not be the first note pattern of the next block.

The notes were continuous sine tones, each with a 250 -msec duration, presented at a level of $70 \mathrm{~dB}$ SPL. The notes were in the same frequency range that was used in Deutsch's (1975) study except that the standard was $A=440$, rather than $A=435$. Notes were digitally generated from one cycle of a sine waveform in an 8K buffer, using the technique of fractional addressing (Hartmann, 1987) controlled by a PDP 11/73 computer. Tones were presented over a pair of headphones (Yamaha YH1000 in Experiments 1 and 2 and Sennheiser HD 480 in Experiment 3).

\section{Procedure}

The listeners were tested individually in a single session lasting 1-1 1/2 h. Each listener was seated in a sound-attenuating room and communicated with the experimenter over an intercom. A listener initiated each trial with a buttonpress. Ten continuous repetitions of a note pattern were then presented. As in Deutsch's (1975) original study, the listener then verbally reported the pattern heard in each ear. The reports were recorded by the experimenter in the next room. This was an open-set report, with no instruction as to what style of reporting the listener should adopt other than that the pattern of notes heard must be fully described. The listener had pencil and paper and, after verbally reporting, was given the opportunity to sketch if so desired.

\section{Results}

\section{Data Treatment}

Two judges, the first author and an individual who was naive as to the purpose of this study, sorted the listeners' data into categories according to the reports for the two ears. Intersorter agreement on this task was $93 \%$. In the few instances where disagreements arose, they were resolved by discussion. The classification categories were as follows: (1) "Arc" responses were considered to be those responses in which the listener reported either a rising then falling or a falling then rising pattern of notes. This was the dominant report in Deutsch's (1975) original experiment and is the response viewed as being consistent with a proximity-based (frequency) organization as found in the scale illusion. (2) "Linear" responses were those in which the listener reported a pattern of notes that either ascended or descended exclusively. This type of report is consistent with a good continuation (trajectory) organization as found in musical scales. Note that in the NS condition this also corresponds to a veridical laterality report. (3) "Jagged" responses were those in which the listener reported a pattern of notes that resembled the pattern of frequencies presented to each ear. For the $D, D+1$, and $D-1$ note patterns this type of response would be consistent with a veridical laterality report. (4) "Other" responses were those reports that did not fit into any of the above three classifications. For instance, a response of a monotone was classified as an "other"
Table 1

Responses to Different Note Patterns (in Percentages) for Experiment 1

\begin{tabular}{lrrrr}
\hline & \multicolumn{4}{c}{ Note Pattern } \\
\cline { 2 - 5 } Response & \multicolumn{1}{c}{$\mathrm{D}$} & $\mathrm{D}+1$ & $\mathrm{D}-1$ & \multicolumn{1}{c}{ NS } \\
\hline Arcs & 67.7 & 49.5 & 46.9 & 6.0 \\
Linear & 21.6 & 39.1 & 37.2 & 90.9 \\
Jagged & 0.0 & 0.0 & 0.0 & 0.0 \\
Other & 10.7 & 11.5 & 15.9 & 3.1 \\
\hline
\end{tabular}

response rather thaר a linear response. This category also included uninterpretable or not-given reports. The results of classification are shown in Table 1.

D note pattern. When presented the original scaleillusion note pattern, the listeners most often gave "arc" responses, which are interpreted as indicating a proximitybased organization. The high prevalence of this type of response is consistent with all other scale-illusion studies. The second most favored responses were "linear" responses, which are interpreted as indicating a good continuation organization. In no case did a listener provide a "jagged" response, which would indicate a lateralitybased organization. Only a small portion of the responses $(10.7 \%)$ fell into the "other" category.

D+1 and D-1 note patterns. These note patterns differed from the original $D$ pattern of the scale illusion in terms of the number of notes. The $D+1$ note pattern was one beat longer, and the $D-1$ note pattern was one beat shorter. As with the D note pattern, the "arc" response was the dominant report. This suggests that the organizational principle operating in the scale illusion, namely, proximity, had the largest influence in these cases as well. However, the percentage of "arc" responses was substantially reduced here, with much of the reduction being taken up by an increase in "linear" responses. (A small portion also went to the "other" category.) There were no "jagged" reports.

NS note pattern. The special feature of the NS note pattern was that both good continuation and laterality cues were allied against proximity cues, which had been shown to dominate them individually. The responses for the NS note pattern indicate that this combination was most persuasive, with the vast majority of responses falling into the "linear" category. This result is consistent with that of Smith et al. (1982), who had also found that listeners could accurately give laterality reports when there was no note switching between ears. These two findings together indicate that while proximity can be a powerful organizer, there are certain circumstances where it does not dominate.

Listener drawings. Six listeners took the opportunity to draw their responses on paper in addition to giving their verbal reports. The drawings largely corresponded to the verbal reports recorded by the experimenter. In a few cases, where the verbal report was ambiguous and the drawing was clear, the drawing was used. Often, however, the drawings appeared to be incomplete. Possibly they were used by the listeners as notes to themselves. 
The results involving listener drawings for this and the following experiments were very few and very similar, so they will not be discussed again.

\section{Data Analysis}

The main question of this experiment was whether the strength of the scale illusion would change with a change in the note pattern that was presented. The strength of the scale illusion is measured by the percentage of proximitybased responses, which becomes operationalized in this data set as the percentage of "arc" responses. The proportion data for the "arc" responses were arcsine transformed and then submitted to a 4 (note pattern) $\times 2$ (origin) $\times 2$ (ear of report) repeated measures analysis of variance (ANOVA). Neither origin nor ear of report had a significant effect on "arc" reports. In contrast, the main effect of note pattern was highly significant $[F(3,45)=$ $30.23, p<.0001]$. Since the NS pattern produced so few "arc" reports, a separate ANOVA was done with data from this condition excluded; the result remained significant $[F(2,30)=5.95, p<.01]$. A $t$ test using the Bonferroni procedure revealed that, with $p<.05$, "arc" reports were more frequent for the $D$ note pattern than for the $\mathrm{D}+1[t(15)=3.16]$ and $\mathrm{D}-1[t(15)=2.62]$ note patterns, which did not differ from one another $(t<1)$. Across the $D, D+1$, and $D-1$ patterns, there was a statistically equivalent rate of "other" responses $(F<1)$. Therefore, the decreases in frequency responses in the $D+1$ and $D-1$ conditions were largely accounted for by increases in good continuation ("linear") responses.

\section{EXPERIMENT 2}

In Experiment 1, good continuation organization was observed as the alternative that emerged when proximitybased organization was reduced. The NS note pattern was overwhelmingly heard as a good continuation organization, and it is possible that its presence may have biased listeners toward giving such reports in general, even on the D note pattern. If the NS note pattern were biasing

\section{RESPONSE ALTERNATIYES}

A)

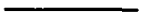

D)

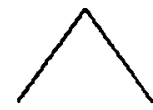

G) $\wedge \wedge$

J) OTHER
B)

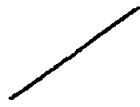

c)

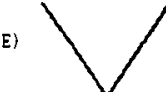

H)

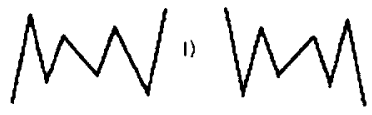

k) DONT KNOW
Figure 3. Listeners' response choices in Experiment 2. Experiment 3 had no "jagged" choices (F-D). Choices F and G corresponded to Pattern D, and choices $H$ and $I$ corresponded to Pattern D+1.
Table 2

Responses to Different Note Patterns (in Percentages) for Experiment 2

\begin{tabular}{lrr}
\hline & \multicolumn{2}{c}{ Note Pattern } \\
\cline { 2 - 3 } \multicolumn{1}{c}{ Response } & \multicolumn{1}{c}{ D } & D+1 \\
\hline Arcs (D \& E) & 40.9 & 17.4 \\
Linear (B \& C) & 11.2 & 22.9 \\
Monotone (A) & 6.5 & 5.7 \\
D jagged (F \& G) & 19.8 & 22.7 \\
D+1 jagged (H \& I) & 12.5 & 23.6 \\
Other (J) & 2.3 & 1.3 \\
Don't know (K) & 6.8 & 6.4 \\
\hline
\end{tabular}

the listeners, then eliminating it could have changed the results. Also in Experiment 1, the verbal reports were written down by the experimenter, which may have introduced an element of experimenter bias.

These bias factors were eliminated in Experiment 2. The procedure in Experiment 2 followed that of Experiment 1 with two notable changes. Experiment 2 included no NS note pattern, and it provided listeners with a closed set of (written) responses (cf. Smith et al., 1982). The closed set is well justified by the finding in Experiment 1 that $90 \%$ of all responses fell into two categories ("arc" and "linear") and that only a small portion fell into the "other" category. Because both the D+ 1 and $D-1$ note patterns produced nearly identical results in Experiment 1, only the $D+1$ note pattern was used here.

\section{Method}

\section{Listeners}

Thirty-two listeners were recruited from the subject pool at Michigan State University and given class credit for their participation. None of the listeners had participated in Experiment 1.

\section{Stimuli and Procedure}

To guide response selection, each listener was presented with a sheet depicting nine alternative patterns that might be heard in each ear. These choices are illustrated in Figure 3 and include: (A) monotone, (B) ascending linear pattern, (C) descending linear pattern, (D) ascending then descending arc pattern, (E) descending then ascending arc pattern, (F) the $D$ jagged pattern, (G) the complimentary $D$ jagged pattern, (H) the $D+1$ jagged pattern, (I) the complimentary D+1 jagged pattern, $(J)$ an "other" option to allow the listener to give responses not consistent with experimenter-provided options, and (K) a "don't know" option to avoid forcing a listener to guess at a response. Each listener heard monaural examples of each of the patterns prior to data collection to ensure that there was an understanding of the manner in which the figures corresponded to the actual sound patterns. If a listener was unsure, further explanation and listening opportunities were given. A listener responded on a sheet with choices running from $A$ through $K$, with each choice corresponding to a particular option. Two columns on the response page allowed the listener to make a different selection for the right and left ears.

\section{Results and Discussion}

The data for Experiment 2 are summarized in Table 2. Consistent with Experiment 1, "arc" responses were more common with the $\mathrm{D}$ note pattern than with the $\mathrm{D}+1$ note pattern. This further demonstrates that a change in the pattern of notes can reduce the strength of the scale 
illusion. In a 2 (note pattern) $\times 2$ (origin) $\times 2$ (ear of report) repeated measures ANOVA, the effect of note pattern was statistically significant $[F(1,31)=16.89, p<$ $.001]$. There were no significant effects involving the origin and ear of report factors. On a separate matter, Experiment 2 showed a reduced rate of good continuation reports for the $\mathrm{D}$ note pattern relative to Experiment 1, suggesting that the NS note pattern may indeed have biased listeners in that experiment.

Another notable finding concerned the $\mathrm{D}$ and $\mathrm{D}+1$ "jagged" responses. In Experiment 2, a large number of responses was of this type and, as a consequence, there was a relatively smaller number of " arc" responses overall. The $D+1$ "jagged" response occurred more often with the D+1 note pattern than with the D note pattern. These data suggest that the listeners may be able to indicate lateral organization of the presented note patterns when given a closed response set. However, there are some considerations that caution against such an interpretation. The presence of a substantial number of veridical responses in a closed set is not an unique finding (see Smith et al., 1982). The scale illusion, while compelling, is not completely convincing. The listeners most likely detected aberrations in the perceived note patterns from time to time and selected more aberrant responses in those cases. In general, the listeners chose the D (Choices $F$ and $G$ ) and D+ 1 (Choices $H$ and I) "jagged" responses equally often. When heard alone, it is difficult to distinguish between the two versions of either matched jagged pattern (e.g., Choice F vs. Choice G), but the difference between the $D$ and $D+1$ jagged patterns is striking (e.g., Choice F vs. Choice $\mathrm{H}$ or I). The increased use of the "jagged" responses with the $\mathrm{D}+1$ note pattern may have happened because the $D$ note pattern is perceptually smoother because of repeated notes at the beginning and end and at the middle. An impression of more complex activity may have biased the listeners to select a more complex response (i.e., F, G, H, or I). The "monotone," "other,", and "don't know" responses occurred infrequently. The combined rate of these responses did not differ for the two note patterns $(F<1)$.

\section{EXPERIMENT 3}

\section{Possible Causes for the Change in} the Strength of the Scale Illusion

\section{Crossing Point}

With the change in the number of notes, there are two features that emerge to distinguish the $D+1$ and $D-1$ note patterns from the $D$ note pattern, either of which might explain the reduced strength of the scale illusion. Consider Figure 4, noting the circled portions in particular. The first feature is the number of notes at the point where the two scales intersect. For the D note pattern, each ear receives a repeated note (either $F$ or $G$ ). The transition from one frequency range into the other in a good continuation organization would be awkward, whereas a separation of streams in a proximity organization is more easily derived. The single note in the middle of the $D+1$ note

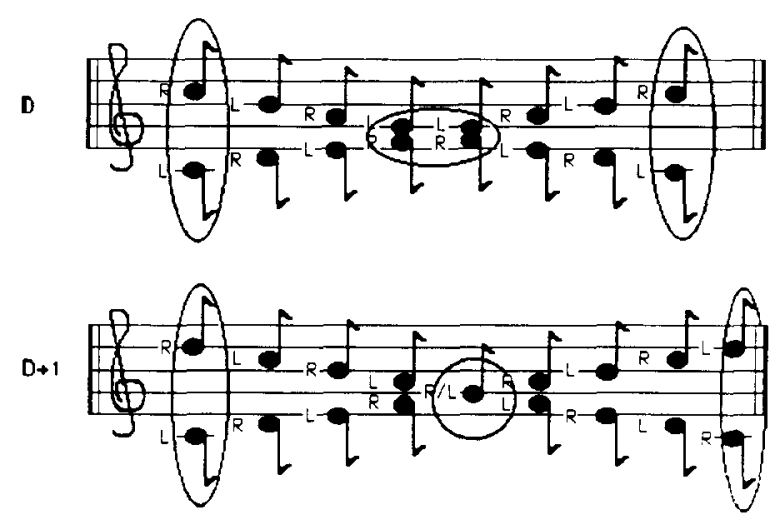

Figure 4. Location of crossing point and first and last note features in Note Patterns $D$ and $D+1$.

pattern is less conducive to a proximity organization. The single note could be considered as an intersection where two scales cross over one another.

\section{First and Last Notes}

The other possibility is that the first and last notes, being located at the extremes of the pitch range, are used as anchor points. The rest of the percept might then be inferred afterward. In the D note pattern, the first and last notes in an ear are either both high Cs or both low $C s$ at the beginning and the end of the sequence. These could provide anchor points in each ear within the same frequency range, thereby supporting a proximity organization. In the $D+1$ note pattern, the first and last notes in a given ear are either initially a high $C$ and finally a low $\mathrm{D}$ or vice versa. These would provide anchor points in each ear in opposite frequency ranges and be less supportive of a proximity organization.

The purpose of Experiment 3 was to choose between the two explanations above: crossing point versus first and last notes. Note patterns possessing only one of the two features were used to test their influence on the scale illusion.

Method
Listeners
Eighteen listeners were recruited during the summer term at
Michigan State University and were paid for their participation.
None of the listeners had participated in either of the previous ex-
periments.

\section{Stimuli and Procedure}

The procedure was identical to Experiment 2 except for the added note patterns, which are illustrated in Figure 5 . In addition to the $D$ and $D+1$ note patterns, four others were included. The Dmod note pattern was identical to the $D$ note pattern with the addition of a note pair at the end. The Dmod-R note pattern was the Dmod note pattern played in reverse order. The $D+1$ mod note pattern was identical to the $D+1$ note pattern except that the final note pair was truncated. The $D+1 \bmod -R$ note pattern was the $D+1$ mod note pattern played in reverse order. The reason for including the Dmod$R$ and $D+1 \bmod -R$ note patterns was that the Dmod and $D+1 \bmod$ note patterns are asymmetrical about the crossing point. The crossing 


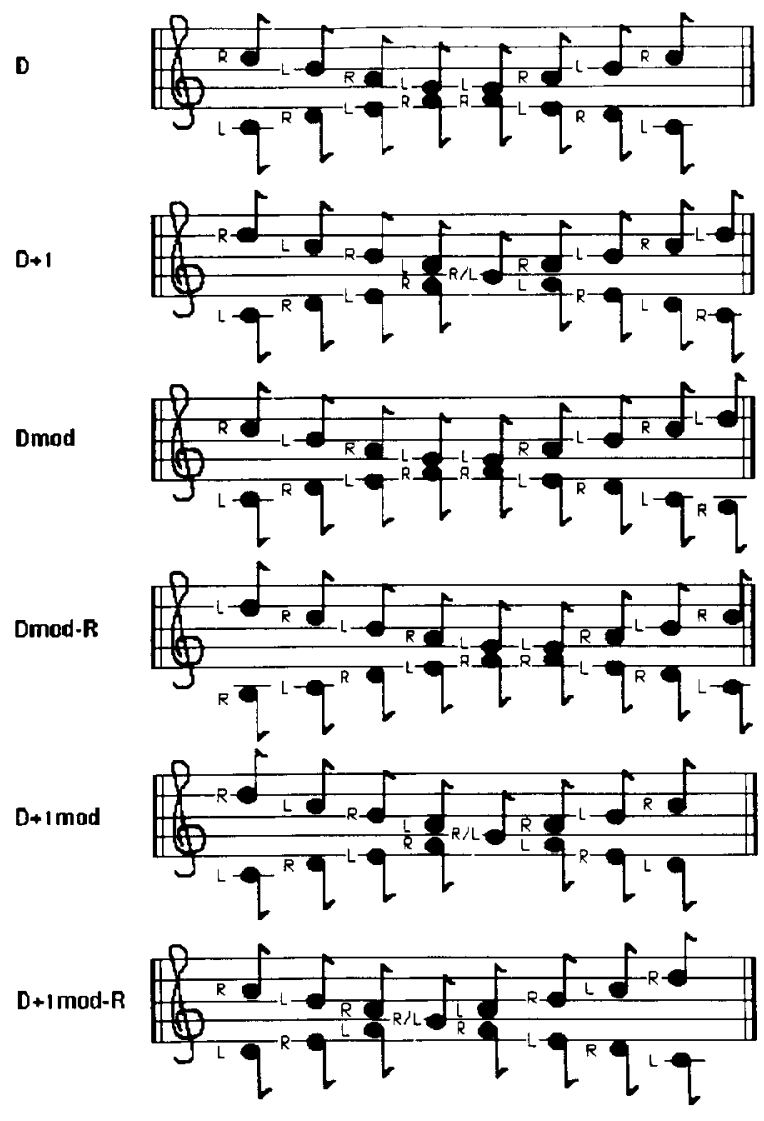

Figure 5. Examples of note patterns presented to listeners in Experiment 3. D has a double-note crossing point and first and last notes in the same frequency range. $D+1$ has a single-note crossing point and first and last notes in different frequency ranges. Dmod has a double-note crossing point and first and last notes in different frequency ranges. D+ 1 mod has a single-note crossing point and first and last notes in the same frequency range. Dmod-R and $D+1 \bmod -R$ are Dmod and D+1mod played in reverse order.

point was further toward the end. The additional note patterns were included to evaluate any possible effects of having a late crossing point. The response sheet was modified to include none of the 12 "jagged" options; there were too many of them.

If the crossing-point feature is critical for construction of a perception, then those note patterns with the single center note $(D+1$, $\mathrm{D}+1 \mathrm{mod}$, and $\mathrm{D}+1 \mathrm{mod}-\mathrm{R}$ ) should result in fewer "arc" reports. Conversely, if the first and last notes are critical, then those note patterns beginning and ending on notes in different pitch ranges for a given ear (D+1, Dmod, and Dmod-R) should result in fewer "arc" reports.

\section{Results and Discussion \\ Response Types \\ The results from this experiment are summarized in Ta-} ble 3 . A 6 (note pattern) $\times 2$ (origin) $\times 2$ (ear of report) repeated measures ANOVA showed a significant difference among the note patterns $[F(5,85)=4.17, p<.01]$. To more fully compare the different response patterns for the different note patterns, in particular whether the crossing point or the first and last notes would play a greater role in disrupting the scale illusion, the effects of each of these properties were considered in turn. There was no significant difference between the double-note crossings ( $D, D m o d$, and Dmod-R) and the single-note crossings $(\mathrm{D}+1, \mathrm{D}+1 \mathrm{mod}$, and $\mathrm{D}+1 \mathrm{mod}-\mathrm{R})$, which both elicited "arc" responses 56\% of the time $(t<1)$. However, those note patterns with first and last notes within the same pitch range $(D, D+1$ mod, and $D+1$ mod-R) elicited "arc" responses $61 \%$ of the time compared with $52 \%$ for those note patterns with first and last notes in different pitch ranges ( $D+1$, Dmod, and Dmod-R), a difference that was statistically significant $[t(17)=2.8$, $p<.05]$. This suggests that the first and last notes have a greater influence over the strength of the scale illusion. The combined rate of "monotone," "other," and "don't know" responses did not differ significantly across the note patterns $(F<1)$. Therefore, differences in the rate of "arc" responses reflected corresponding differences in the rate of "linear" responses. There were no significant effects involving either the origin or ear of report factors.

\section{GENERAL DISCUSSION}

The present set of experiments extends the findings of investigations of the scale illusion (Butler, 1979, Deutsch, 1975,1982 ; Smith et al., 1982) by showing that the organization of complex arrays of musical notes is not done exclusively on the basis of proximity (frequency range). Instead, if the pattern of notes is altered slightly, listeners' proximity organizations diminish and other organizations, in particular a good continuation organization, emerge. To our knowledge, these are the only experiments to find such a result by varying note structure. However, it should be noted that Smith et al. were able to weaken the scale illusion by altering tone intensities and timbres, leading listeners to select good continuation-based, but not laterality-based, patterns.

On the one hand, as noted in the introduction, the scaleillusion finding was to be anticipated on the basis of other

Table 3

Responses to Different Note Patterns (in Percentages) for Experiment 3

\begin{tabular}{lrrrccc}
\hline & \multicolumn{5}{c}{ Note Pattern } \\
\cline { 2 - 7 } \multicolumn{1}{c}{ Response } & \multicolumn{1}{c}{ D } & D +1 & Dmod & Dmod-R & D +1 mod & D+1 mod-R \\
\hline Arcs (D \& E) & 67.1 & 53.0 & 50.9 & 50.9 & 57.6 & 58.1 \\
Linear (B \& C) & 15.3 & 28.0 & 26.4 & 30.3 & 25.7 & 27.1 \\
Monotone (A) & 10.4 & 4.4 & 5.8 & 3.5 & 7.6 & 4.6 \\
Other (J) & 2.1 & 5.3 & 6.0 & 7.4 & 2.8 & 4.6 \\
Don't know (K) & 5.1 & 9.3 & 10.9 & 7.9 & 6.3 & 5.6 \\
\hline
\end{tabular}


studies that showed the importance of proximity/frequency range (e.g., Bregman \& Campbell, 1971; Tougas \& Bregman, 1987, 1990). On the other hand, as Deutsch (1975) herself points out, the illusion was more surprising when compared with dichotic listening studies that have been done with linguistic materials (e.g., Broadbent, 1954; Cherry, 1953; Moray, 1959; Treisman, 1961). In that domain, listeners find it easy to report either the phonetic or semantic content of a speech stream presented to one ear while ignoring competing input to the other ear. Additionally, when the information in both ears is attended to, reports are generally given on a "by-source" basis, rather than on a "by-order-of-input" basis (Broadbent, 1954). If the input can be organized into semantically meaningful wholes that are constantly switching ears, then semantically ordered output is preferred (Gray \& Wedderburn, 1960). If this were a generalizable auditory phenomenon, then the listeners in a scale-illusion study should report the note patterns presented to each ear (Figure 1a) or a meaningful pattern such as a musical scale (Figure 1c). Least likely would be the frequency-based organization that they seem to prefer.

On another issue, it has been noted that listeners in scale-illusion experiments sometimes report hearing a pattern of notes that moves from ear to ear (Deutsch, 1982). The closed set of responses given in Experiments 2 and 3 did not included this possibility since it would have directly violated the task, namely, to identify and report the pattern of notes in each ear. This presents a difficulty in interpreting the data from such experiments; however, in our case the listeners did not complain about limitations of the response set.

There are still some unanswered questions relating to the scale illusion. Some of these questions concern whether listeners reporting good continuation responses heard a crossing of two patterns (e.g., Tougas \& Bregman, 1987, 1990), how knowledge of musical scales might affect the perception of the figure, and, based on recent evidence (Deutsch, 1991), what influence the nature of the vertical (harmonic) structure might have on perceptual organization. Additionally, more work needs to be done to properly assess the role of salient features such as notes at the extremes of the pitch range and at the crossing point.

\section{REFERENCES}

Bregman, A. S., Campbell, J. (1971). Primary auditory stream segregation and perception of order in rapid sequences of tones. Journal of Experimental Psychology, 89, 244-249.

BrondBENT, D. E. (1954). The role of auditory localization in attention and memory span. Joumal of Experimental Psychology, 47, 191-196.

ButLer, D. (1979). A further study of melodic channeling. Perception \& Psychophysics, 25, 264-268.

CherRy, E. C. (1953). Some experiments on the recognition of speech, with one and with two ears. Journal of the Acoustical Society of America, 25, 975-979.

Deutsch, D. (1974). An auditory illusion. Nature, 251, 307-309.

DeuTsch, D. (1975). Two-channel listening to musical scales. Journal of the Acoustical Society of America, 57, 1156-1160.

Deutsch, D. (1982). Grouping mechanisms in music. In D. Deutsch (Ed.), The psychology of music (pp. 99-134). Orlando: Academic Press.

Deutsch, D. (1991). Pitch proximity in the grouping of simultaneous tones. Music Perception, 9, 185-198.

DowLING, W. J. (1968). Rhythmic fission and the perceptual organization of tone sequences. Unpublished doctoral dissertation, Harvard University, Cambridge.

DowlING, W. J. (1973). The perception of interleaved melodies. Cognitive Psychology, 5, 322-337.

Gray, J. A., WedDerburn, A. A. I. (1960). Grouping strategies with simultaneous stimuli. Quarterly Joumal of Experimental Psychology, 12, 180-184.

HARTMANN, W. M. (1987). Digital waveform generation by fractional addressing. Journal of the Acoustical Society of America) 82, 1883-1891.

HartmanN, W. M., \& Johnson, D. (1991). Stream segregation and peripheral channeling. Music Perception, 9, 155-183.

MorAy, N. (1959). Attention in dichotic listening: Affective cues and the influence of instructions. Quarterly Joumal of Experimental Psychology, 11, 56-60.

Smith, J., Hausfield, S., Power, R. P., Gorta, A. (1982). Ambiguous musical figures and auditory streaming. Perception \& Psychophysics, 32, 454-464.

Treisman, A. M. (1961). Contextual cue in selective listening. Quarterly Journal of Experimental Psychology, 13, 242-248.

Tougas, Y., \& Bregman, A. S. (1987). Crossing of auditory streams. Journal of Experimental Psychology: Human Perception \& Performance, 11, 788-798.

Tougas, Y., Bregman, A. S. (1990). Auditory streaming and the continuity illusion. Perception \& Psychophysics, 47, 121-126.

(Manuscript received April 29, 1991; revision accepted for publication March 2, 1992.) 